\title{
Segmentation of Bright Targets Using Wavelets and Adaptive Thresholding
}

\author{
Xiao-Ping Zhang, Member, IEEE, and Mita D. Desai
}

\begin{abstract}
A general systematic method for the detection and segmentation of bright targets is developed in this paper. We use the term "bright target" to mean a connected, cohesive object which has an average intensity distribution above that of the rest of the image. We develop an analytic model for the segmentation of targets, which uses a novel multiresolution analysis in concert with a Bayes classifier to identify the possible target areas. A method is developed which adaptively chooses thresholds to segment targets from background, by using a multiscale analysis of the image probability density function (PDF). A performance analysis based on a Gaussian distribution model is used to show that the obtained adaptive threshold is often close to the Bayes threshold. The method has proven robust even when the image distribution is unknown. Examples are presented to demonstrate the efficiency of the technique on a variety of targets.
\end{abstract}

Index Terms-Adaptive thresholding, Bayes classifier, image segmentation, multiresolution analysis, target detection, wavelet transforms.

\section{INTRODUCTION}

$\mathbf{M}$ ANY methods for segmenting objects of interest (which we generically refer to as targets) from a noisy, cluttered background have been developed [1]-[5]. Among these, many involved greyscale-based thresholding strategies [5]-[8], which usually assume the image to have a uniform and stationary, or at least quasi-stationary, distribution of intensities over the target and over the background. Such methods are not very effective when the images have complex, nonstationary distributions of intensities. It is usually difficult to adapt global techniques by simply using local processing, since the resulting operators are usually more sensitive to noise [9] and generally yield desultory improvement.

However, if the image is observed at a particular scale, or range of scales, viz., by passing it through a bandpass filter, the resulting image intensity distribution will often exhibit a greater degree of local stationarity. In this way, the performance of local adaptive segmentation/thresholding algorithms can be improved.

\footnotetext{
Manuscript received March 27, 1997; revised April 3, 2001. This work was supported in part by NIH Grant GM07717, NASA Grant NAG10-0155, Texas Advanced Research Program Grant ARP002-1996, and Canada NSERC Grant RGPIN239031. The associate editor coordinating the review of this manuscript and approving it for publication was Dr. Dapang Chen.

$\mathrm{X}$.-P. Zhang is with the Department of Electrical and Computer Engineering, Ryerson Polytechnic University, Toronto, ON, M5B 2K3 Canada (e-mail: xpzhang@ieee.org).

M. Desai is with the Division of Engineering, University of Texas, San Antonio, TX 78249 USA.

Publisher Item Identifier S 1057-7149(01)05435-5.
}

Wavelet transform-based methods offer a natural framework for providing multiscale image representations that can be separately analyzed [10], [11]. For example, through a multiscale decomposition, most of the gross intensity distribution can be isolated in a large scale image, while the information about details and singularities, such as edges and textures, can be isolated in mid- to small scales.

In this paper, a new and systematic method for the segmentation of potential target areas is presented based on a particular wavelet decomposition and a Bayes classifier. In Section II, an adaptive multiscale threshold selection criterion is developed which analyzes the image probability density function (PDF). In Section III, a statistical analysis of the performance of the adaptive threshold selection strategy is presented. In Section IV, several segmentation examples are given for a variety of interesting images, including a mammogram and a FLIR image. Section V discusses the results and concludes the paper.

\section{Segmentation of Possible Targets}

\section{A. Multiscale Bayes Approach to Target Segmentation}

The term bright target is used to mean a connected, cohesive object which has an average intensity distribution above that of the rest of the image. Such targets usually appear as having a blob-like appearance. Of course, other image components will occur that are bright, but which may be poorly connected or which occur only over a small scale. Using a natural multiscale decomposition, such as an appropriate wavelet transform, coherent targets may be separated from, and subsequently distinguished from, transient bright objects.

We begin by exploring a simple approach to target segmentation that involves isolating the putative target scale through the selection of an appropriate scale in the wavelet decomposition, then thresholding the single-scale image using a Bayes classifier. The method is used as a benchmark comparison for the method proposed in Section II-B. The Bayes approach, while optimal in a statistical sense, requires certain a priori information and suffers from some limitations.

The normal discrete wavelet transform (DWT) [12]-[14], can be computed by the pyramidal algorithm

$$
\begin{aligned}
\nu_{n}^{0} & =x[n] \\
\nu_{n}^{j} & =\sum_{k} \nu_{k}^{j-1} g[k-2 n], \quad j=1,2, \ldots \\
w_{n}^{j} & =\sum_{k} \nu_{k}^{j-1} h[k-2 n], \quad j=1,2, \ldots
\end{aligned}
$$

where $g$ and $h$ are the analysis scaling and wavelet filters, respectively. It is easy to generalize the above one-dimensional 


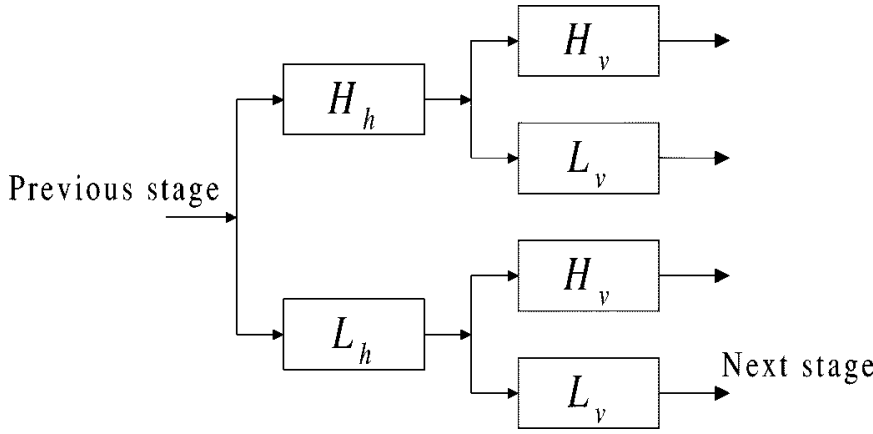

Fig. 1. One stage of 2-D wavelet decomposition.

(1-D) transform to two-dimensional (2-D) transform. One stage of 2-D wavelet decomposition by subband filter can then be described in Fig. 1. There is one scaling channel $\left(L_{h} L_{v}\right)$ and there are three wavelet channels $\left(H_{h} H_{v}, H_{h} L_{v}\right.$, and $\left.L_{h} H_{v}\right)$.

The normal decimated DWT has a very sparse sampling grid which is desirable for coding and compression applications but often not suitable for signal analysis applications [15], [12]. Therefore, the undecimated DWT is used in the presented method. The undecimated DWT can also be computed by fast cascade filter algorithms [13].

In the large scale image components $I^{(j)}, j=1,2, \ldots$, the high-frequency singularities disappear and the distribution of masses is smoothed.

The segmentation of possible targets can be modeled by the following classification problem. For an ideal image $I(m, n)$, there are pixels belonging to two classes: 1) the background $C_{b}$ and 2) the target $C_{t}$. The segmentation of possible targets then becomes a problem of choosing one of the two hypotheses:

$$
\begin{aligned}
& H_{1}: I(m, n) \in C_{b} \\
& H_{2}: I(m, n) \in C_{t} .
\end{aligned}
$$

Assume $p_{I}(x)$ is the PDF of image $I$. Then

$$
p_{I}(x)=P\left(C_{b}\right) p_{b}(x)+P\left(C_{t}\right) p_{t}(x)
$$

where

$p_{b}(x) \quad$ PDF of the class $C_{b}$;

$P\left(C_{b}\right)$ a priori probability of class $C_{b}$ in image $I$;

$p_{t}(x) \quad$ PDF of the class $C_{t}$;

$P\left(C_{t}\right)$ a priori probability of class $C_{t}$ in image $I$.

The above classification problem is solved by the standard Bayes classifier

$$
f_{b}(x) \stackrel{H_{1}}{\gtrless} f_{t}(x)
$$

where

$$
f_{b}(x) \triangleq p_{b}\left(x / C_{b}\right) P\left(C_{b}\right)
$$

and

$$
f_{t}(x) \triangleq p_{t}\left(x / C_{t}\right) P\left(C_{t}\right)
$$

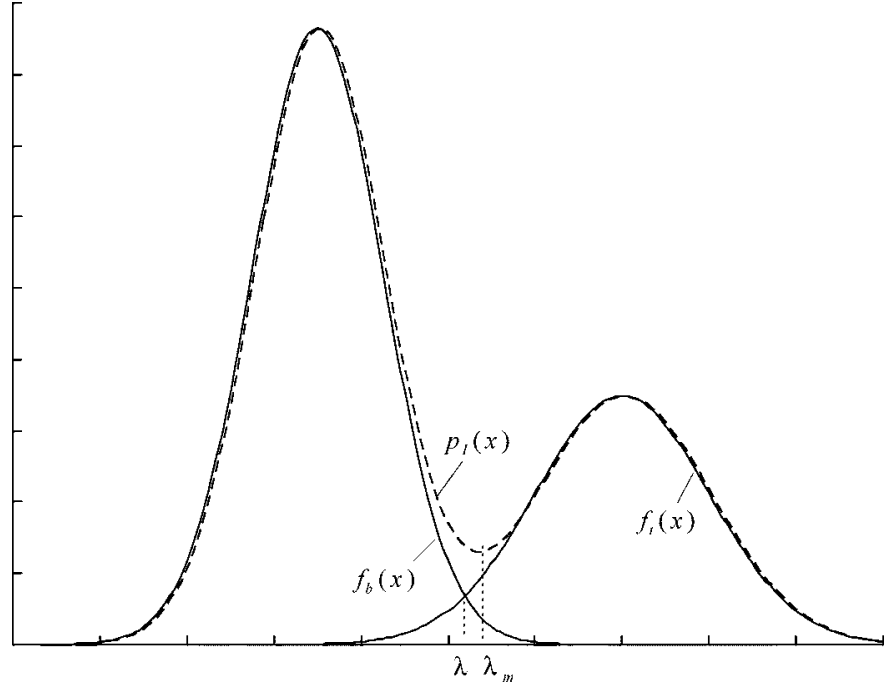

Fig. 2. Dashed line indicates the PDF $p_{I}(x)$. Two solid lines indicate $P\left(C_{b}\right) p_{b}(x)$ and $P\left(C_{t}\right) p_{t}(x)$, respectively. The Bayes threshold $\lambda$ and the proposed threshold $\lambda_{m}$ are indicated.

Assuming that $f_{b}(x)$ and $f_{t}(x)$ have one point of intersection $\lambda$, as illustrated in Fig. 2, the above classifier is equivalent to the following threshold detection criterion:

$$
\left\{\begin{array}{l}
I(m, n)<\lambda: I(m, n) \in C_{b} \\
I(m, n) \geq \lambda: I(m, n) \in C_{t}
\end{array}\right.
$$

where $\lambda$ is chosen so that

$$
f_{b}(\lambda)=f_{t}(\lambda)
$$

A segmented image at scale $j$ using the Bayes classifier can be expressed as

$$
I_{\text {seg }}^{(j)}(m, n)=\left\{\begin{array}{ll}
1, & I^{(j)}(m, n)>\lambda \\
0, & I^{(j)}(m, n)<\lambda
\end{array} .\right.
$$

There are two main problems in using the above Bayes classifier for segmentation of targets in an image. First of all, images may have a rather complex structure; they contain other classes. For exampl, in a mammogram, the object of segmentation is the tumor which generally has a higher greyscale value than the remainder of the tissue. Within a mammogram, there are calcifications, dense tissue, and various kinds of noise, etc., in addition to the normal tissues and possible tumors (targets). The normal tissues and possible targets also contain some singular pixels. Hence, many pixels may be misclassified by using simple threshold detection method on original mammograms.

Second, in practice, we do not actually know the PDF of any class as well as the a priori probability of every class. In addition, the probability distributions often vary greatly in different images so that a general distribution cannot be obtained to describe all images.

By analyzing the image at a coarser scale, singularities can be removed. However, the PDF of any class as well as the a priori probability are still unknown in the low-resolution images. It is difficult to choose a suitable threshold automatically. Of course, trial and error is a possible approach. However, it is inefficient and difficult to use in practice. In the following, a method based 
on wavelet analysis is developed to find an appropriate threshold adaptively and automatically for different images.

\section{B. Choosing Threshold Using Wavelet Analysis}

Recall that the image PDF (5) is determined by class PDFs and the $a$ priori probability of every class in it.

When the PDF $p_{I}(x)$ is known, but the class PDFs and the a priori probabilities of the two classes are unknown, the following method is presented to choose the threshold.

In the new method, the threshold is selected as the "global" local minimum point, which is shown as $\lambda_{m}$ in Fig. 2. Note that here the global local minimum point means the minimum point of the PDF at a larger scale. For the PDF $p_{I}(x)$ in (5), we have

$$
\frac{\partial p_{I}(x)}{\partial x}=P\left(C_{b}\right) \frac{\partial p_{b}(x)}{\partial x}+P\left(C_{t}\right) \frac{\partial p_{t}(x)}{\partial x} .
$$

Then $\lambda_{m}$ will satisfy the following:

$$
\left.\frac{\partial p_{I}(x)}{\partial x}\right|_{x=\lambda_{m}}=0
$$

and

$$
\left.\frac{\partial^{2} p_{I}(x)}{\partial x^{2}}\right|_{x=\lambda_{m}}>0 .
$$

Comparing it with the Bayes threshold in (8), it can be seen that $\lambda_{m}$ is different, although they may be very close in many cases.

Because the PDFs and the a priori probabilities of the two classes are unknown, and the PDF of every image does not have a specified distribution, it is impossible to calculate the ideal Bayes threshold. Therefore, the proposed new method can usually find a very good threshold which can be reasonably treated as an estimation of the optimal Bayes threshold.

The image PDF $p_{I}(x)$ can be easily computed as the image histogram. Using a multiscale analysis, we have removed the singularities in the image and made it suitable for target segmentation using a threshold classifier. However, there are usually many fluctuations in the PDFs of the images. In practice, there will be many local minima in PDF curves. Obviously, what we want to find is the global local minimum point, i.e., the local minimum point at a large scale. Again, the wavelet transform can be used to analyze the PDF of the image and to find the global local minimum point. To obtain a better localization of the threshold, once again we suggest the use of the undecimated DWT.

In the new approach, the wavelet transformed curves of the PDF at every scale are aligned according to the maximum of them. In general, the threshold at a certain scale of PDF is chosen by looking for the largest local minimum. One can also find the local minimum at several scales of PDF, and then obtain a weighted average as the final threshold for the target segmentation problem.

In the presented new method, a scheme to adaptively select the scale of 1-D wavelet transform is developed. Let $I_{\text {seg }}^{(j)}$ denote the segmented areas in the wavelet transformed image at scale $j$ by the selected threshold. The segmentation $I_{\text {seg }}^{(j)}$ for the possible targets will normally be a sparse binary matrix. The portion of the segmented target areas should be in a reasonable range, i.e., the size of the segmented targets should be in some reasonable range. Therefore, if the segmentation $I_{\text {seg }}^{(j)}$ which is obtained using the threshold found in a certain scale is not reasonable, we should try to find a new threshold in a coarser scale. This can be done easily because the wavelet transform value at the coarser scale can be calculated efficiently and quickly from the value at the finer scale by cascaded fast filter algorithms [13], and the calculations for segmented areas $I_{\text {seg }}^{(j)}$ are simple binary operations.

Next, we find the corresponding areas of the segmentation in the original image. Using the selected threshold, we can obtain the segmented areas $I_{\text {seg }}^{(j)}$, which represent the possible targets, in the wavelet transformed image at scale $j$. However, there will be some spatial shift in the target location in the transformed image compared with the original image because of the time delay $L_{d}$ of the FIR wavelet filters. Actually, one pixel in the wavelet transformed image represents a piece of original image, because the image is analyzed by a bank of windows in wavelet transform. The sizes of the windows vary in different scales. This point should be taken into account when the segmented areas are mapped to the original image.

In the dyadic DWT, assume the lengths of both the scaling filter $H$ and the wavelet filter $G$ are $L$. Since the overall scaling filter and wavelet filter at scale $j$ should be $\prod_{i=0}^{j-1} \sqrt{2^{i}} H\left(z^{2^{i}}\right)$ and $G\left(z^{2^{j-1}}\right) \prod_{i=1}^{j-2} \sqrt{2^{i}} H\left(z^{2^{i}}\right)$, respectively, it is easy to show that the lengths of the overall scaling filter and wavelet filter are $L_{j}=\left(2^{j}-1\right) L-2^{j}+2$. Hence, one pixel of image at scale $j$ stands for an area with at most size $L_{j} \times L_{j}$ in the original image, and the position of the pixel is the center of this area. The adjusted segmented area $I_{\text {seg }}$ in the original image using the segmented area $I_{\text {seg }}^{(j)}$ at scale $j$ is shown in (12) at the bottom of the page.

In this way, an attempted segmentation of the target at each scale can be obtained. It is also possible to define a weighted combination of them to obtain the final segmented area depending on the scales of interest. A block diagram of the above wavelet-based adaptive segmentation method is shown in Fig. 3.

Sometimes, several large scale global local minima of the image PDF can be obtained, because there may be more than one class of bright targets. If only two classes are expected, the largest global local minimum should be chosen as the threshold for the segmentation of possible target areas since it is the most

$$
I_{\text {seg }}(m, n)= \begin{cases}1, & \text { if } I_{\text {seg }}^{(j)}(k, l)=1 \text { and } \\ & (m, n) \in\left[\left(k-L_{d}, l-L_{d}\right),\left(k-L_{d}+L_{j}-1, l-L_{d}+L_{j}-1\right)\right] \\ 0, & \text { otherwise }\end{cases}
$$




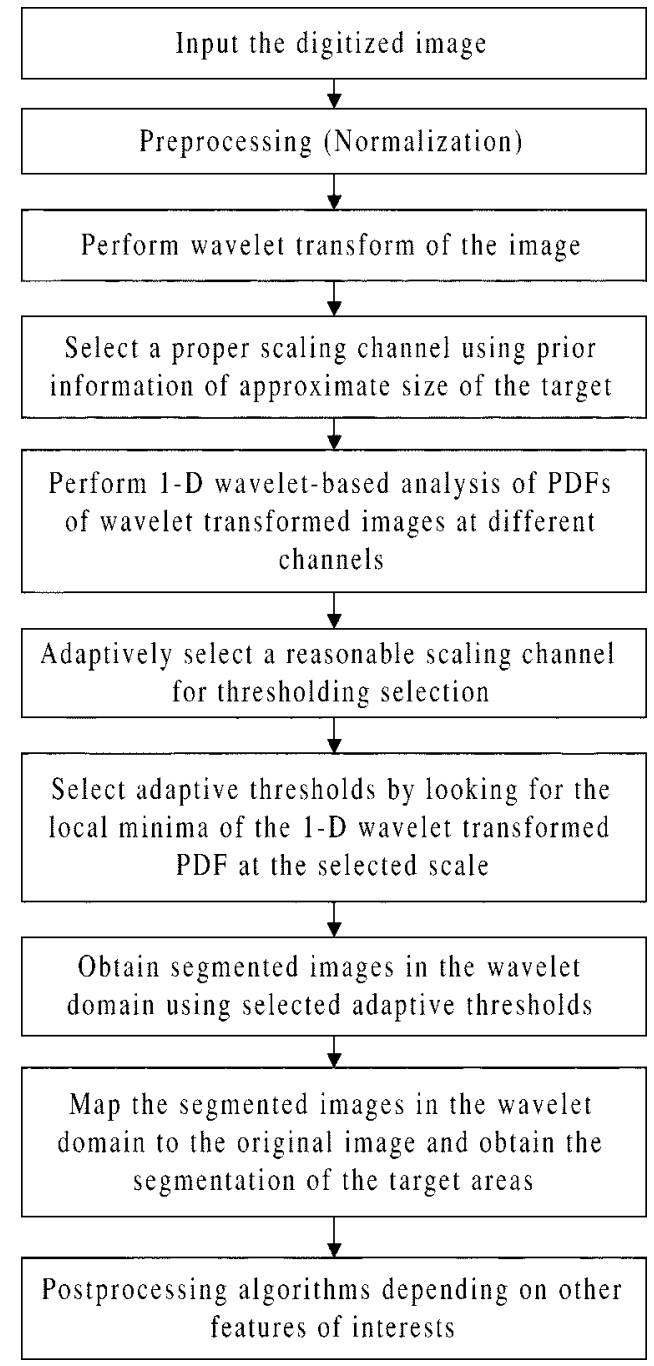

Fig. 3. Block diagram of the adaptive segmentation method.

abnormal. That is, assume $\Lambda$ is the set of $M$ local minima of the PDF, i.e.,

$$
\Lambda=\left\{\lambda_{i}\left|\frac{\partial p_{I}(x)}{\partial x}\right|_{x=\lambda_{i}}=0 \text { and }\left.\frac{\partial^{2} p_{I}(x)}{\partial x^{2}}\right|_{x=\lambda_{i}}>0\right\} .
$$

Then without loss of generality, assume

$$
\lambda_{1}<\lambda_{2}<\cdots<\lambda_{M}
$$

In our method, the adaptive threshold $\lambda_{m}$ is selected as the largest local minimum, i.e.,

$$
\lambda_{m}=\max _{i}\left\{\lambda_{i} \mid \lambda_{i} \in \Lambda, i=1, \ldots, M\right\}=\lambda_{M} .
$$

It is also possible to treat the segmented image as a new "original" image, which may include more detailed classes. Then, a wavelet analysis of the PDF can be used again to classify the different classes within the segmented area. The process can be iterated again until certain criteria are satisfied.

\section{Performance of the Method Under Gaussian DISTRIBUTIONS}

It is well known that the wavelet transform is a type of linear transform. A pixel value in the transformed image is actually some linear combination of $P \times P$ pixels in the neighborhood of this pixel, i.e.,

$$
y(m, n)=\mathbf{w}_{v} \mathbf{X} \mathbf{w}_{h}
$$

where $y(m, n)$ denotes a pixel of transformed image, the $P \times$ $P$ matrix $\mathbf{X}$ denotes a matrix of input pixels, $\mathbf{w}_{v}$ denotes the vertical wavelet transform vector, and $\mathbf{w}_{h}$ denotes the horizontal wavelet transform vector.

Normally, the input pixels in a neighborhood should belong to the same class and can be treated as independent identically distributed (i.i.d.) random variables with the same distribution. Then according to the central-limit theorem [16], as $P$ increases, the distribution of $y(m, n)$ approaches a Gaussian distribution. Therefore, we can approximately treat the gray level of each class in the transformed image as having a Gaussian distribution.

Hence, the performance of the presented method will be analyzed based on the Gaussian distribution. Assume both classes in transformed image are Gaussian distributed, i.e.,

and

$$
p_{1}(x)=\frac{1}{\sqrt{2 \pi} \sigma_{1}} \exp \left\{-\frac{\left(x-m_{1}\right)^{2}}{2 \sigma_{1}^{2}}\right\}
$$

$$
p_{2}(x)=\frac{1}{\sqrt{2 \pi} \sigma_{2}} \exp \left\{-\frac{\left(x-m_{2}\right)^{2}}{2 \sigma_{2}^{2}}\right\} \text {. }
$$

Then, for a Bayes threshold $\lambda$, we have

$$
\begin{aligned}
& P\left(C_{1}\right) \frac{1}{\sqrt{2 \pi} \sigma_{1}} \exp \left\{-\frac{\left(\lambda-m_{1}\right)^{2}}{2 \sigma_{1}^{2}}\right\} \\
& \quad=P\left(C_{2}\right) \frac{1}{\sqrt{2 \pi} \sigma_{2}} \exp \left\{-\frac{\left(\lambda-m_{2}\right)^{2}}{2 \sigma_{2}^{2}}\right\}
\end{aligned}
$$

i.e.,

$$
\frac{P\left(C_{1}\right)}{P\left(C_{2}\right)} \cdot \frac{\sigma_{2}}{\sigma_{1}}=\exp \left\{\frac{\left(\lambda-m_{1}\right)^{2}}{2 \sigma_{1}^{2}}-\frac{\left(\lambda-m_{2}\right)^{2}}{2 \sigma_{2}^{2}}\right\} .
$$

For simplification, first assume $m_{1}<m_{2}$ without loss of generality. Then take $P_{C}=\left(P\left(C_{1}\right) / P\left(C_{2}\right)\right)$ and $\sigma=\left(\sigma_{2} / \sigma_{1}\right)$. Normalize with respect to class $C_{1}$, i.e., let $t=\left(\left(x-m_{1}\right) / \sigma_{1}\right)$ and denote $m_{d}=\left(\left(m_{2}-m_{1}\right) / \sigma\right)$. Equation (18) can be rewritten as

$$
\ln D=\frac{\lambda_{1}^{2}}{2}-\frac{\left(\lambda_{1}-m_{d}\right)^{2}}{2 \sigma^{2}},
$$

where $D=P_{C} \cdot \sigma$ and $\lambda_{1}=\left(\left(\lambda-m_{1}\right) / \sigma_{1}\right)$. From the above equation, it can be seen that $\lambda_{1}$ is only of concern to $P_{C}, \sigma$, and $m_{d}$.

Obviously, only the solution of $\lambda_{1}$ for the above equation in the range $\left[0, m_{d}\right]$ should be taken. The solutions which are out of the range $\left[0, m_{d}\right]$ are meaningless for the classification of two classes. 
Assume $P\left(C_{1}\right)$ and $P\left(C_{2}\right)$ are the a priori probabilities of class $C_{1}$ and $C_{2}$ in image $I$. Then (5) can be rewritten as

$$
p_{I}(x)=f_{1}(x)+f_{2}(x)
$$

where $f_{1}(x) \triangleq p_{1}(x) P\left(C_{1}\right)$ and $f_{2}(x) \triangleq p_{2}(x) P\left(C_{2}\right)$.

For the proposed threshold $\lambda_{m}$, we have

$\left.\frac{\partial p_{I}(x)}{\partial x}\right|_{x=\lambda_{m}}=\left.\frac{\partial f_{1}(x)}{\partial x}\right|_{x=\lambda_{m}}+\left.\frac{\partial f_{2}(x)}{\partial x}\right|_{x=\lambda_{m}}=0$

and

$\left.\frac{\partial p_{I}^{2}(x)}{\partial x^{2}}\right|_{x=\lambda_{m}}=\left.\frac{\partial^{2} f_{1}(x)}{\partial x^{2}}\right|_{x=\lambda_{m}}+\left.\frac{\partial^{2} f_{2}(x)}{\partial x^{2}}\right|_{x=\lambda_{m}}>0$

Substituting (15) and (16) into (21), we get

$$
\begin{aligned}
& P\left(C_{1}\right) \cdot\left\{\frac{\lambda_{m}-m_{1}}{\sigma_{1}^{3}} \cdot \exp \left(-\frac{\left(\lambda_{m}-m_{1}\right)^{2}}{2 \sigma_{1}^{2}}\right)\right\} \\
& \quad+P\left(C_{2}\right) \cdot\left\{\frac{\lambda_{m}-m_{2}}{\sigma_{2}^{3}} \cdot \exp \left(-\frac{\left(\lambda_{m}-m_{2}\right)^{2}}{2 \sigma_{2}^{2}}\right)\right\}=0 .
\end{aligned}
$$

Also, it can be rewritten as

$$
\begin{aligned}
P_{C} & \cdot\left\{\sigma \cdot \lambda_{m 1} \cdot \exp \left(-\frac{\lambda_{m 1}^{2}}{2}\right)\right\} \\
& +\left\{\frac{\lambda_{m 1}-m_{d}}{\sigma} \cdot \exp \left(-\frac{\left(\lambda_{m 1}-m_{d}\right)^{2}}{2 \sigma^{2}}\right)\right\}=0
\end{aligned}
$$

where $\lambda_{m 1}=\left(\left(\lambda_{m}-m_{1}\right) / \sigma_{1}\right)$. It can be seen that $\lambda_{m 1}$ is only of concern to $P_{C}, \sigma$, and $m_{d}$. That means the probability of misclassification error is only of concern to $P_{C}, \sigma$, and $m_{d}$, because the probability of misclassification error of class $C_{1}$ is

$$
\begin{aligned}
P_{E 1}\left(\lambda_{m 1}\right) & =P\left(C_{1}\right) \cdot P\left\{H_{2} / x \in C_{1}\right\} \\
& =P\left(C_{1}\right) \cdot \operatorname{erfc}_{*}\left(\lambda_{m 1}\right)
\end{aligned}
$$

and the probability of misclassification error of class $C_{2}$ is

$$
\begin{aligned}
P_{E 2}\left(\lambda_{m 1}\right) & =P\left(C_{2}\right) \cdot P\left\{H_{1} / x \in C_{2}\right\} \\
& =P\left(C_{2}\right) \cdot \operatorname{erfc}_{*}\left(\frac{\lambda_{m 1}-m_{d}}{\sigma}\right)
\end{aligned}
$$

where the function $\operatorname{erfc}_{*}(x)$ is defined as [17]

$$
\operatorname{erfc}_{*}(x) \triangleq \int_{x}^{\infty} \frac{1}{\sqrt{2 \pi}} \exp \left(-\frac{x^{2}}{2}\right) d x
$$

It is easy to see that when $\lambda_{m 1}$ is out of the range $\left[0, m_{d}\right]$, it cannot be used for the classification of two classes.

It is difficult to solve the nonlinear equation (24). To obtain an estimate of $\lambda_{m}$, the Taylor series expansion is employed at point $\lambda$

$$
\begin{aligned}
f_{1}(x)= & f_{1}(x-\lambda)+\left.f_{1}^{\prime}(x)\right|_{x=\lambda} \cdot(x-\lambda) \\
& +\left.f_{1}^{\prime \prime}(x)\right|_{x=\lambda} \cdot(x-\lambda)^{2}+\cdots
\end{aligned}
$$

and

$$
\begin{aligned}
f_{2}(x)= & f_{2}(x-\lambda)+\left.f_{2}^{\prime}(x)\right|_{x=\lambda} \cdot(x-\lambda) \\
& +\left.f_{2}^{\prime \prime}(x)\right|_{x=\lambda} \cdot(x-\lambda)^{2}+\cdots
\end{aligned}
$$

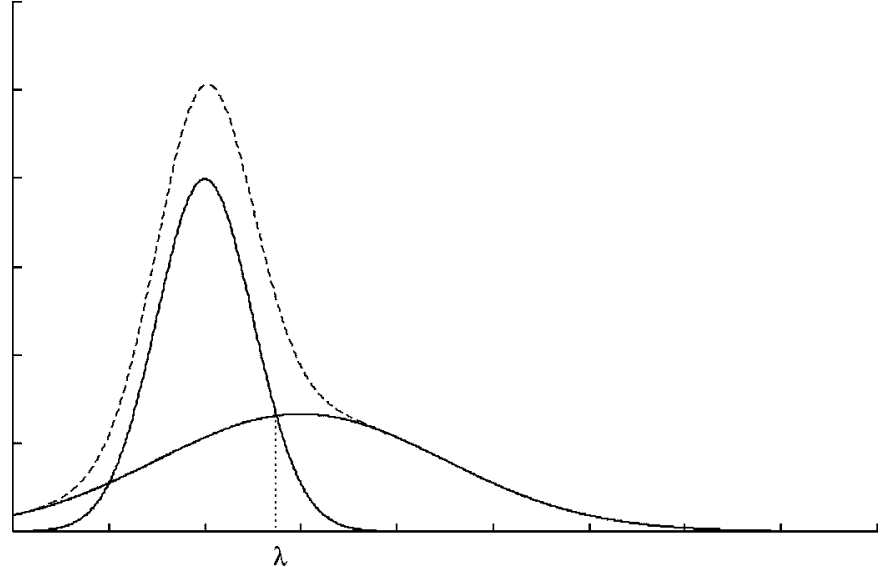

Fig. 4. Dashed line indicates the PDF $p_{I}(x)$. Two solid lines indicate $P\left(C_{1}\right) p_{1}(x)$ and $P\left(C_{2}\right) p_{2}(x)$, respectively. The Bayes threshold $\lambda$ is indicated.

We take the terms with order less than three. Substituting (15) and (16) into the above equation, and using (18) and (21), we obtain

$$
\lambda_{m} \approx \lambda+\frac{\sigma_{2}\left(\frac{\lambda-m_{1}}{\sigma_{1}}\right)-\sigma_{1}\left(\frac{m_{2}-\lambda}{\sigma_{2}}\right)}{\frac{\sigma_{2}}{\sigma_{1}} \cdot\left(\frac{\lambda-m_{1}}{\sigma_{1}}\right)^{2}+\frac{\sigma_{1}}{\sigma_{2}} \cdot\left(\frac{\lambda-m_{2}}{\sigma_{2}}\right)^{2}-\left(\frac{\sigma_{2}}{\sigma_{1}}+\frac{\sigma_{1}}{\sigma_{2}}\right)} .
$$

Similarly, it can be rewritten as

$$
\Delta \lambda_{1} \approx \frac{\sigma \lambda_{1}-\left(\frac{m_{d}-\lambda_{1}}{\sigma}\right)}{\sigma\left(\lambda_{1}^{2}-1\right)+\frac{1}{\sigma} \cdot\left[\left(\frac{m_{d}-\lambda_{1}}{\sigma}\right)^{2}-1\right]}
$$

where

$$
\Delta \lambda_{1} \triangleq \frac{\lambda_{m}-\lambda}{\sigma_{1}} .
$$

It can also be obtained from (22) that

$\left.\frac{\partial p_{I}^{2}(x)}{\partial x^{2}}\right|_{x=\lambda_{m}} \approx \sigma\left(\lambda_{1}^{2}-1\right)+\frac{1}{\sigma} \cdot\left[\left(\frac{m_{d}-\lambda_{1}}{\sigma}\right)^{2}-1\right]>0$.

Equation (31) shows that $m_{d}$ must be large enough to choose the local minimum point as the threshold, i.e., $\lambda_{1}>1$ and $\left(\left(m_{d}-\lambda_{1}\right) / \sigma\right)>1$. In fact, if (31) is not satisfied, the two classes will have a large overlap so that the misclassification error will be large even using the Bayes threshold. Fig. 4 shows an example.

From these equations, it is easy to see that when $m_{d}$ becomes larger, $\lambda_{1}$ becomes larger and then $\Delta \lambda_{1}$ will become smaller. However, $\lambda_{1}$ and $\Delta \lambda_{1}$ have no simple and monotonic relationship with $\sigma$ and $P_{C}$. In the following figures, the performance of the threshold in different cases is given.

Because of the symmetry of the Gaussian function, we can assume $P_{C}>1$ without loss of generality.

In Fig. 5, the curves show how the threshold and its performance change with different $\sigma$ and $P_{C}$ but constant $m_{d}$, where $\sigma$ ranges in $[0.1,10], P_{C}=2^{n}, n=1,2, \ldots, 7$, and $m_{d}=5$. 


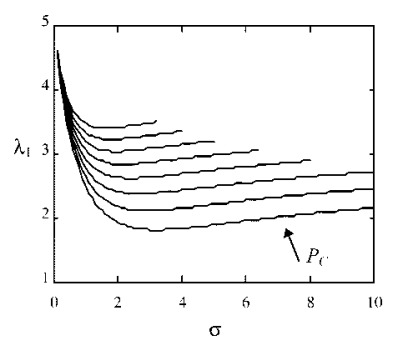

(a)

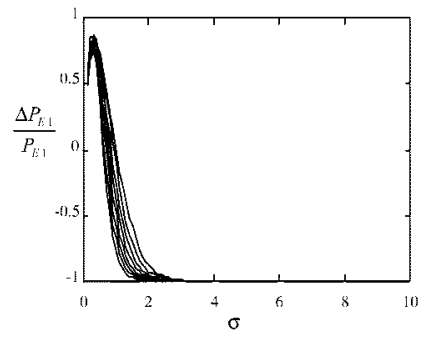

(c)

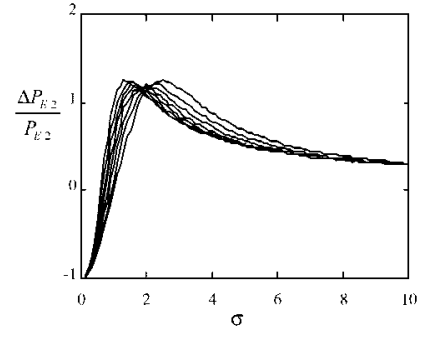

(e)

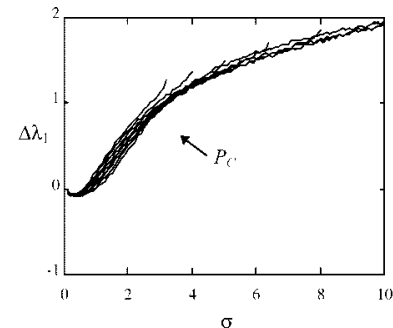

(b)

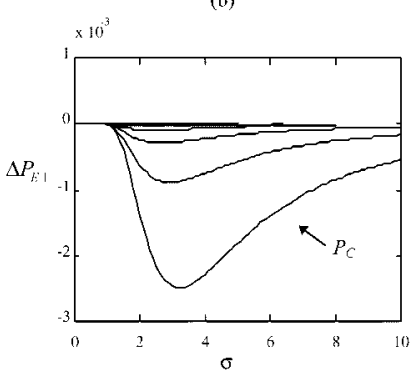

(d)

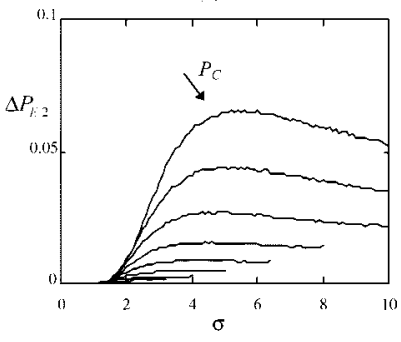

(f)

Fig. 5. Performance of the proposed threshold with respect to $\sigma$ and $P_{C}$. The different curves stand for different $P_{C}=2^{n}, n=1,2, \ldots, 7$, while $m_{d}=5$. (a) The Bayes threshold $\lambda_{1}$. (b) The difference $\Delta \lambda_{1}$ between the proposed threshold $\lambda_{m 1}$ and the Bayes threshold $\lambda_{1}$. (c) The relative error of error probability $P_{E 1}$ between the proposed threshold $\lambda_{m 1}$ and the Bayes threshold $\lambda_{1}$. (d) The absolute error of $P_{E 1}$ between $\lambda_{m 1}$ and $\lambda_{1}$. (e) The relative error of error probability $P_{E 2}$ between $\lambda_{m 1}$ and $\lambda_{1}$. (f) The absolute error of $P_{E 2}$ between $\lambda_{m 1}$ and $\lambda_{1}$.

Fig. 5(a) shows the Bayes threshold $\lambda_{1}$. The different curves stand for different $P_{C}$. Clearly, $\lambda_{1}$ increases as $P_{C}$ increases, but does not monotonically vary with $\sigma$. Fig. 5(b) shows the difference $\Delta \lambda_{1}$ between the threshold $\lambda_{m 1}$ and the Bayes threshold $\lambda_{1}$. Now $\Delta \lambda_{1}$ varies greatly as $\sigma$ varies, but varies little as $P_{C}$ changes. As we would expect from (22) and (31), some curves do not range completely over $[0.1,10]$ of $\sigma$ because the local minima do not exist in $\left[0, m_{d}\right]$ in these cases when $\sigma$ is greater than some $\sigma_{0}$.

In Fig. 5(c) and (d), the differences of the error probability of misclassification $P_{E 1}$ for class $C_{1}$ between $\lambda_{m 1}$ and $\lambda_{1}$ are plotted. Fig. 5(c) shows the relative error $\left(\left(P_{E 1}\left(\lambda_{m 1}\right)-P_{E 1}\left(\lambda_{1}\right)\right) / P_{E 1}\left(\lambda_{1}\right)\right)$ and Fig. 5(d) shows the absolute error $P_{E 1}\left(\lambda_{m 1}\right)-P_{E 1}\left(\lambda_{1}\right)$. From the figures, it can be seen that the relative error of $P_{E 1}$ is less than one for the proposed threshold. And the absolute error of $P_{E 1}$ is still rather small when the relative error is large.

Similar things happen for $P_{E 2}$. As shown in Fig. 5(e) and (f), the relative error and absolute error of $P_{E 2}$ are plotted, respectively. The relative error of $P_{E 2}$ is less than 1.5 for our threshold. The absolute error of $P_{E 2}$ is larger than that of $P_{E 1}$ because we have a relatively larger $P_{E 2}\left(\lambda_{1}\right)$ even for the Bayes threshold when $\sigma$ is larger. An example is shown in Fig. 6.

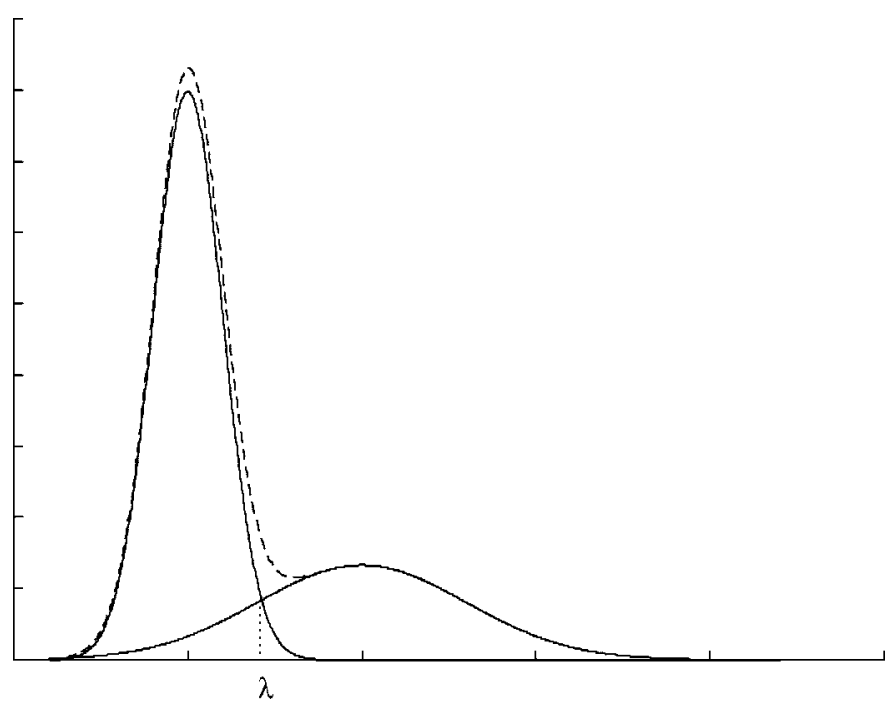

Fig. 6. Dashed line indicates the PDF $p_{I}(x)$. Two solid lines indicate $P\left(C_{1}\right) p_{1}(x)$ and $P\left(C_{2}\right) p_{2}(x)$, respectively. We have a relatively larger $P_{E 2}(\lambda)$ for the Bayes threshold $\lambda$.

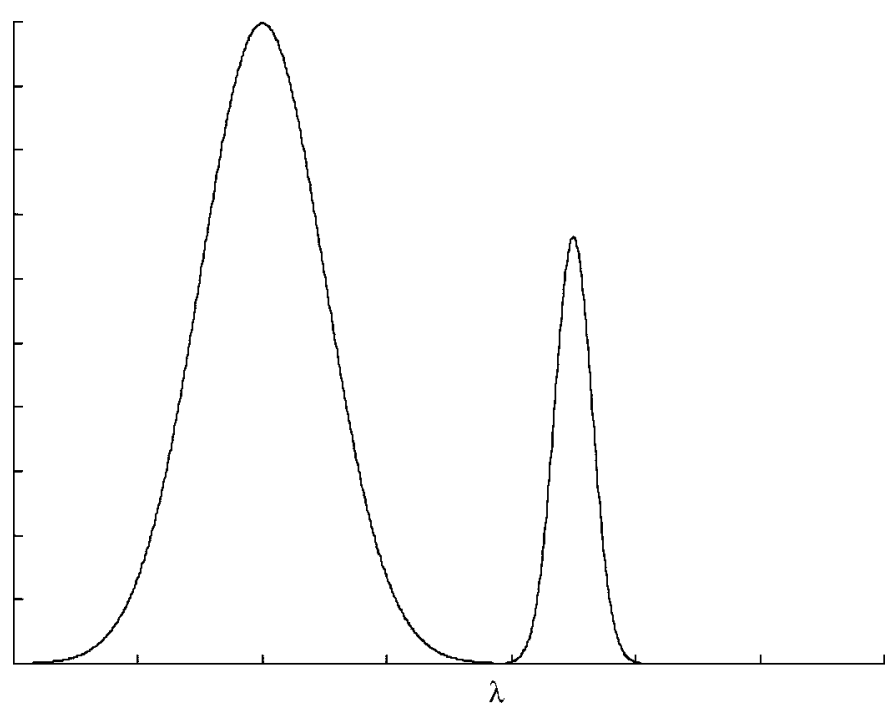

Fig. 7. Dashed line indicates the PDF $p_{I}(x)$. Two solid lines indicate $P\left(C_{1}\right) p_{1}(x)$ and $P\left(C_{2}\right) p_{2}(x)$, respectively. When $\sigma$ is very small, $\Delta \lambda_{1}$ also becomes very small. Note that the dashed line and two solid lines almost overlap completely.

It is also shown that when $P_{C}$ increases, the errors for both $P_{E 1}$ and $P_{E 2}$ decrease, because class $C_{2}$ has less influence for the whole image. When $\sigma$ is very small, it can be seen that $\Delta \lambda_{1}$ and the error also become very small. This is because the class $\mathrm{C}_{2}$ is more concentrated so that the distance between two classes [18] becomes larger when $m_{d}$ is constant. Hence it is easier to distinguish two classes. This can also be expected from (30). Fig. 7 shows the PDFs in these cases.

In Fig. 8, the performance of the proposed threshold is given with $\sigma$ and $m_{d}$ varying and constant $P_{C}$, where $\sigma$ ranges over $[0.1,10], m_{d}=2 n-1, n=1,2, \ldots, 9$, and $P_{C}=5$.

The same things are shown in Fig. 8(a)-(f) as in Fig. 5(a)-(f). In Fig. 8(a), it can be seen that $\lambda_{1}$ increases monotonically as $m_{d}$ increases. This can be expected in (19). From Fig. 8(b), it is shown that $\Delta \lambda_{1}$ decreases when $m_{d}$ increases, as can be expected. Also, when $m_{d}$ is smaller or $\sigma$ is larger, the distance 

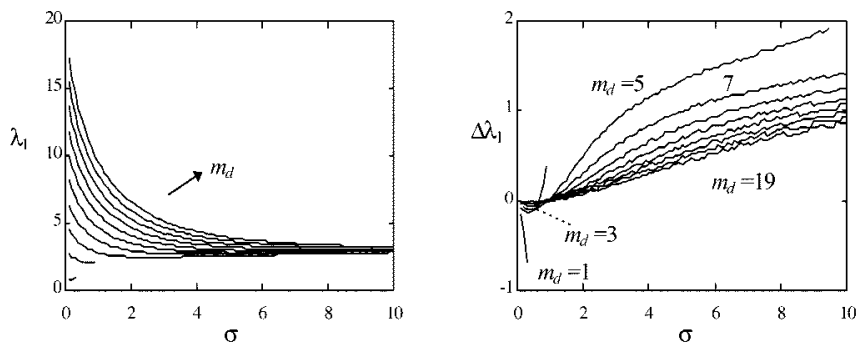

(a)

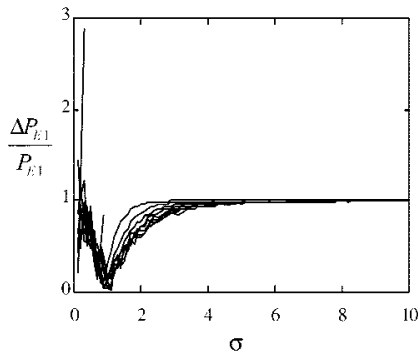

(c)

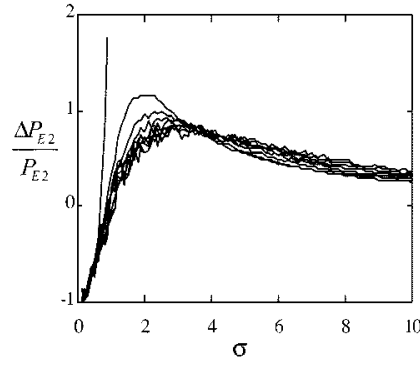

(e)

(d)

(f)
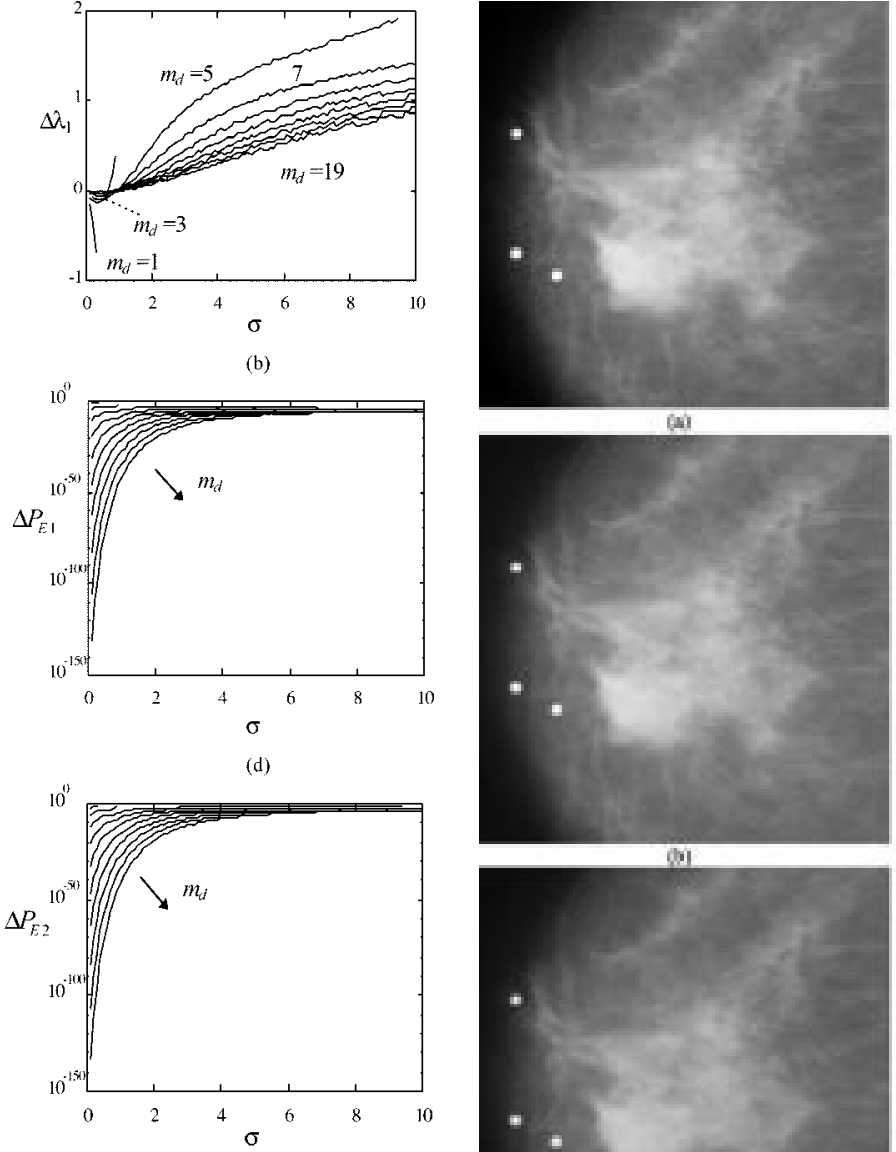

Fig. 8. Performance of the proposed threshold with respect to $\sigma$ and $m_{d}$. The different curves indicate different $m_{d}=2 n-1, n=1,2, \ldots, 9$, while $P_{C}=5$. (a) The Bayes threshold $\lambda_{1}$. (b) The difference $\Delta \lambda_{1}$ between the proposed threshold $\lambda_{m 1}$ and the Bayes threshold $\lambda_{1}$. (c) The relative error of error probability $P_{E 1}$ between the proposed threshold $\lambda_{m 1}$ and the Bayes threshold $\lambda_{1}$. (d) The absolute error of $P_{E 1}$ between $\lambda_{m 1}$ and $\lambda_{1}$. (e) The relative error of error probability $P_{E 2}$ between $\lambda_{m 1}$ and $\lambda_{1}$. (f) The absolute error of $P_{E 2}$ between $\lambda_{m 1}$ and $\lambda_{1}$.

between the two classes will be smaller. Then it is more difficult to distinguish between the two classes. Hence, the local minima may not exist in $\left[0, m_{d}\right]$ when $\sigma$ is greater than some $\sigma_{0}$ for some $m_{d}$. Therefore, some curves in Fig. 8(b) do not span the entire range of $\sigma,[0.1,10]$.

In Fig. 8(c) and (e), it can be seen that the relative errors of $P_{E 1}$ and $P_{E 2}$ between the proposed threshold and the Bayes threshold are not very large and vary little when $m_{d}$ changes. It is shown in Fig. 8(d) and (f) that the absolute errors of $P_{E 1}$ and $P_{E 2}$ are very small and decrease monotonically as $m_{d}$ increases.

From the above analysis, it can be seen that for Gaussian distributions, the proposed threshold can give a rather good performance in a reasonable range of parameters. It is obvious that the more "separable" the two classes are, the closer performance to the Bayes threshold the proposed threshold has.

Of course, the larger is $P$, the closer the distribution of $y(m, n)$ approaches the Gaussian distribution and the less noise remains, however, the more space localization of the classes may be lost. We should get tradeoff when selecting the scale for segmentation.

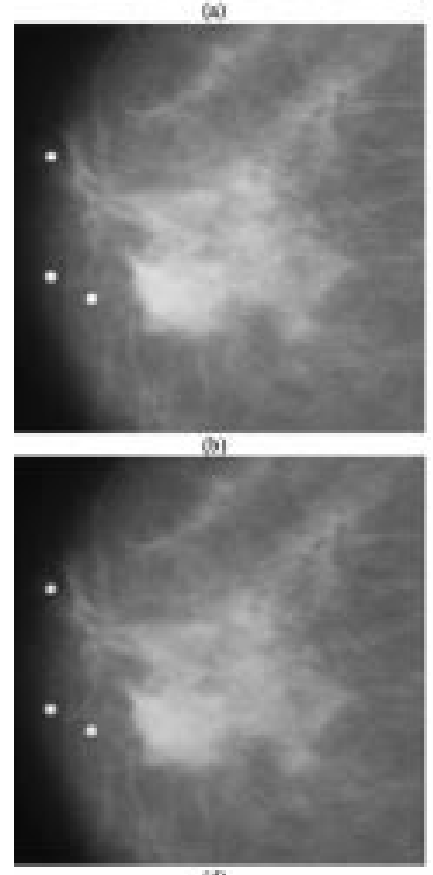

(ब)
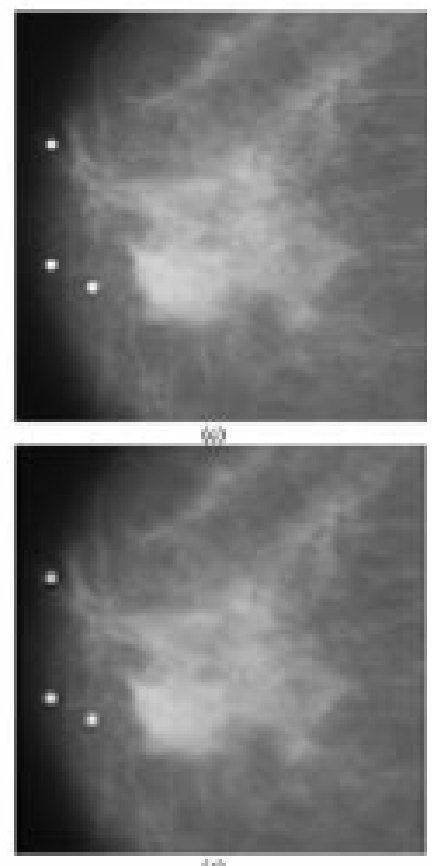

(4)

Fig. 9. (a) Original $1024 \times 1024$ mammogram image for a real patient. (b)-(e) Images of four scaling channels of DWT.

When the distribution is not Gaussian, taking terms with order less than three in the Taylor series equations (27) and (28), and using (21), we have

$$
\Delta \lambda \triangleq \lambda_{m}-\lambda \approx-\frac{f_{1}^{\prime}(\lambda)+f_{2}^{\prime}(\lambda)}{f_{1}^{\prime \prime}(\lambda)+f_{2}^{\prime \prime}(\lambda)}
$$

which means $\Delta \lambda$ will be small when $f_{1}^{\prime \prime}(\lambda)$ and $f_{2}^{\prime \prime}(\lambda)$ have large values. Larger values of $f_{1}^{\prime \prime}(\lambda)$ and $f_{2}^{\prime \prime}(\lambda)$ can be expected when the two classes are both concentrated. Usually, the object we want to segment has a relative high concentration. For example, the target areas in the mammogram are often such classes. Therefore, the proposed threshold is often close to the Bayes threshold in these cases. In practical image segmentation problems, usually the Bayes threshold cannot be calculated because the PDFs and a priori probability are unknown. In the proposed threshold, only a few loose and reasonable assumptions are used. Hence, the robust performance can be expected in the new method.

\section{EXAMPLES}

In the following, we would like to give additional examples to show the applications of the new method. 

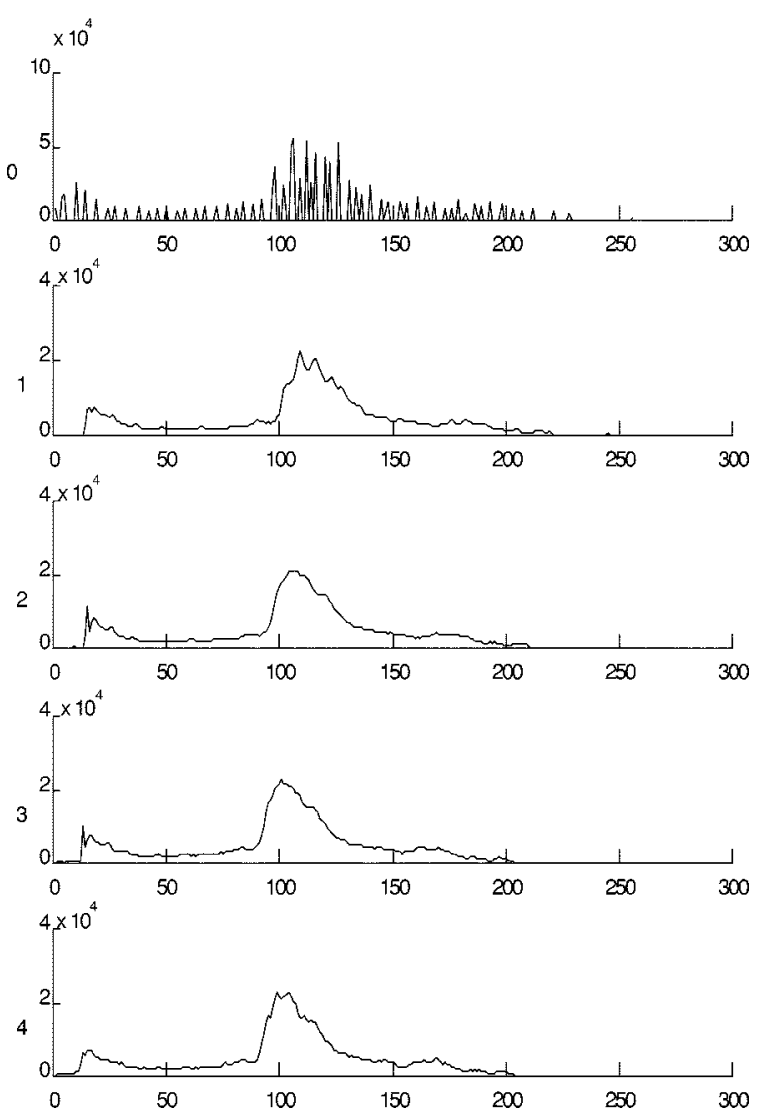

Fig. 10. Histograms (PDFs) of the four transformed images in Fig. 9(b)-(e). The top one shows the histogram of the original image.

The first example is a $1024 \times 1024$ mammogram image with 256 greyscale and $100 \mu \mathrm{m} \times 100 \mu \mathrm{m} / \mathrm{pixel}$, an overview of the breast of a real patient. The original image is shown as Fig. 9(a). Daubechies 6-point wavelet [11] is selected to process the image. Then the $L_{h} L_{v}$ scaling channel will mainly maintain the greyscale information of blobs of size about $6 \times 6$. Fig. 9(b)-(e) shows the $L_{h} L_{v}$ parts of its wavelet transforms from scales 1 to 4. Fig. 10 indicates the histograms (PDFs) of these four transformed images. The image in scale 2 is used for segmentation. For the second step, Daubechies 6-point wavelet [11] is used again for analysis of PDF of the wavelet transformed image because of its performance in denoising [19]. Next, we take 5-scale wavelet transforms for the histogram of the image in scale 2. They are illustrated as Fig. 11. By taking the local minima of the curve at adaptively selected scale, four local minima, $\lambda_{1}<\lambda_{2}<\lambda_{3}<\lambda_{4}$, are obtained. Using the largest local minimum, $\lambda_{4}$, as the adaptive threshold, the segmented areas are shown in Fig. 12(a). Fig. 12(b) shows the masked image by $I_{\text {seg }} \cdot I+I$, which enhances the segmented areas. It can be seen that these segmented areas are just lead markers used by radiologists. It is also shown that the segmented areas contain the areas of interest. By using $\lambda_{3}$, the tumor area is obtained, which is shown in Fig. 13(a) and (b). For $\lambda_{2}$ and $\lambda_{1}$, almost all tissue areas are segmented from the background. Fig. 14(a) and (b) show the segmented areas using $\lambda_{2}$. The segmented areas by $\lambda_{1}$ have more transition areas than that by $\lambda_{2}$.

In this example, Daubechies 6-point wavelet is used and scale 2 is selected for classification. It is an appropriate size for the ap-

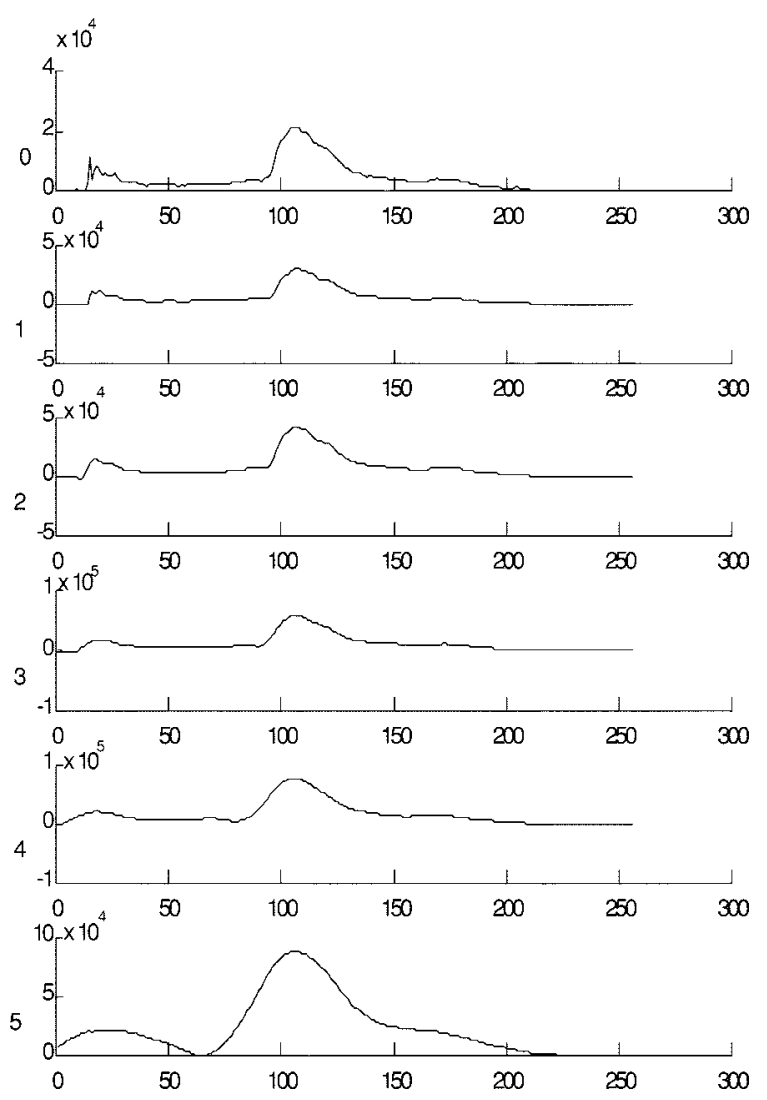

Fig. 11. Five-scale wavelet transform for the histogram of the image in scale 2 [Fig. 9(c)].
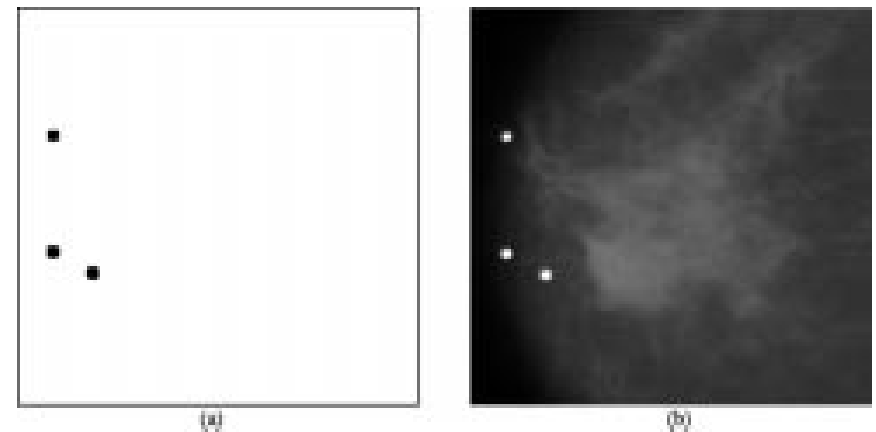

Fig. 12. (a) Segmented areas using $\lambda_{4}$. (b) The masked image using the segmented image $I_{\text {seg }}$ in (a) by $I_{\text {seg }} \cdot I+I$.
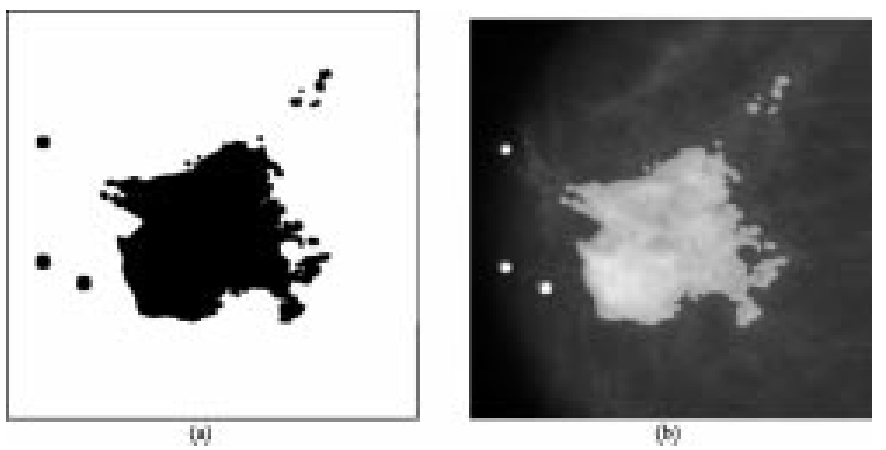

Fig. 13. (a) Segmented areas using $\lambda_{3}$. (b) Masked image using the segmented image $I_{\text {seg }}$ in (a) by $I_{\text {seg }} \cdot I+I$.

plication because in this case, $L_{2}=17$ and the wavelet window size is $17 \times 17$. In this scale, it can be expected that most noise 


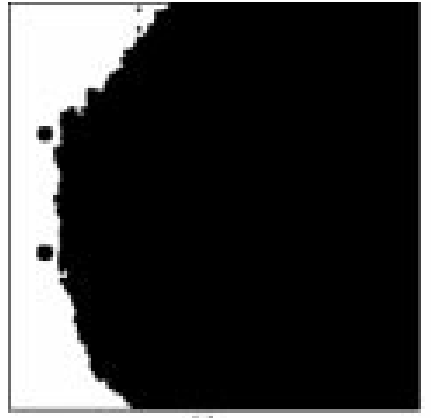

(a)

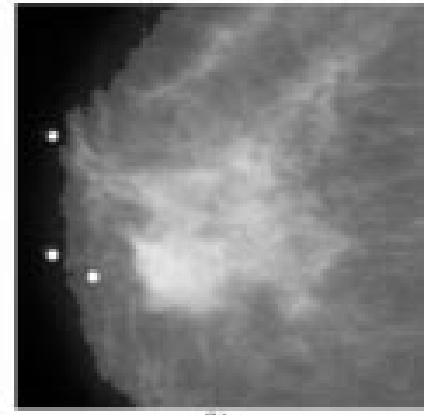

(b)
Fig. 14. (a) Segmented areas using $\lambda_{2}$. (b) Masked image using the segmented image $I_{\text {seg }}$ in (a) by $I_{\text {seg }} \cdot I+I$.

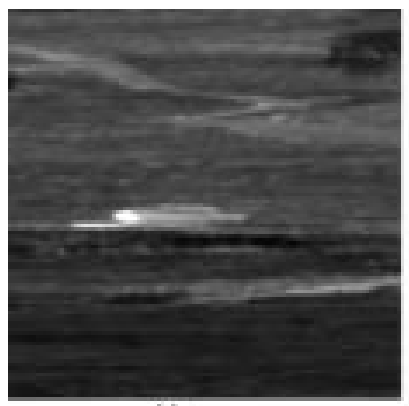

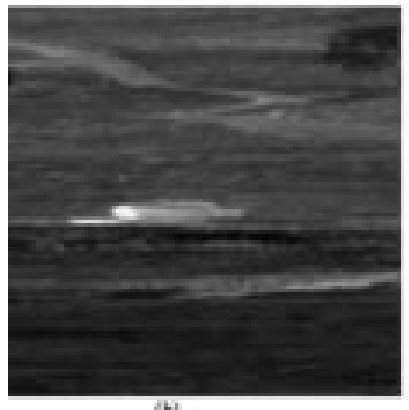

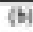

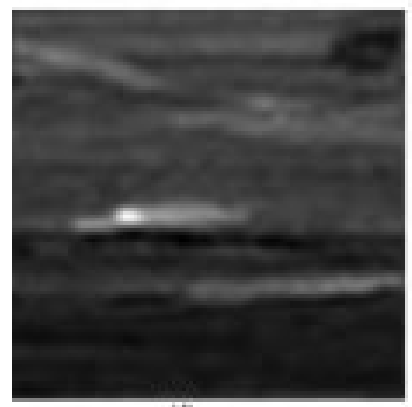

(1)

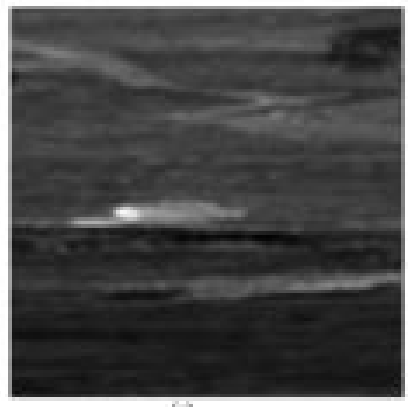

(E)

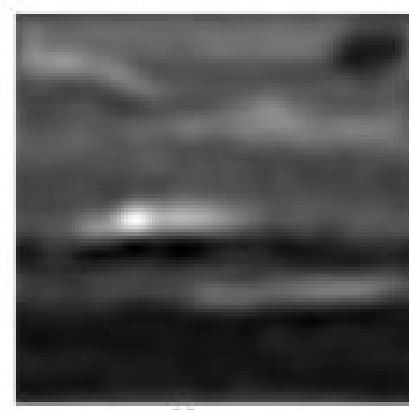

(c)
Fig. 15. (a) A $256 \times 256$ infrared image about a tank. (b)-(e) Images of four scaling channels of DWT.

and singularities can be suppressed by dropping the coefficients of the wavelet channels, and the bright target-tumor information remains in the scaling channel. In PDF analysis, we still selected Daubechies 6-point wavelet because of its good performance in noise removal [19]. We not only want to remove the noise from the PDF curve, but also want to remove the fluctuation within the curve. Then, a rather large scaling channel 4 is selected adaptively so that most of the local minima disappear and only those global local minima remain.
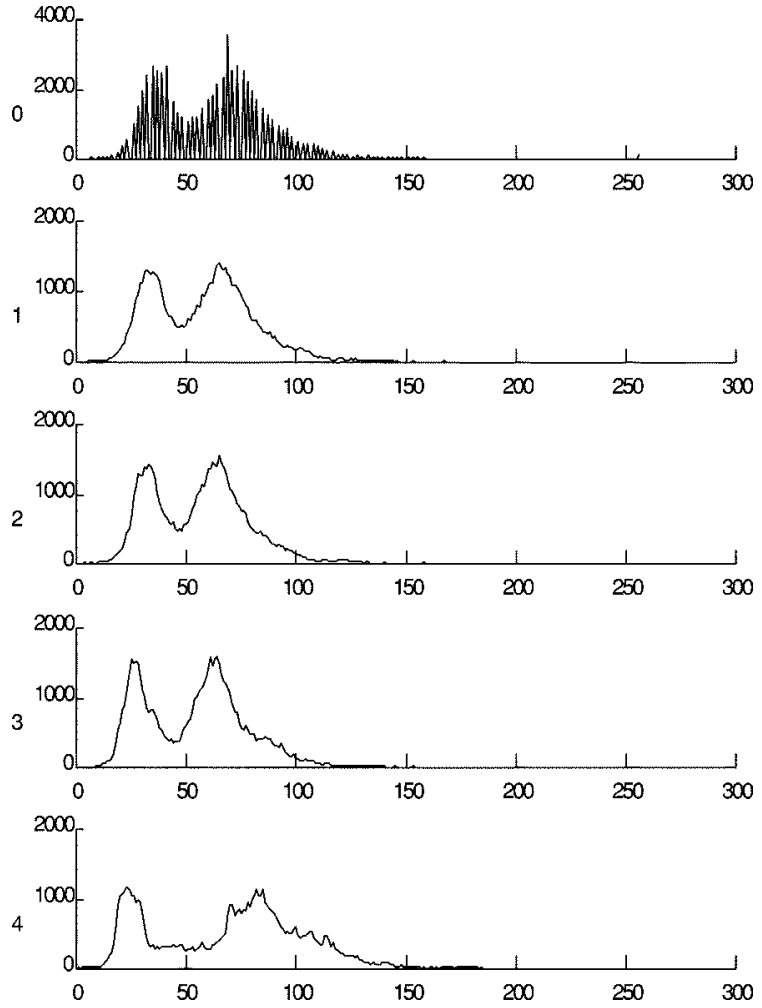

Fig. 16. PDFs of the four transformed images in Fig. 15(b)-(e). The top one shows the histogram of the original image.

As would be expected in this example, the wavelet transform on the original image removed the singularities and obtained the mass greyscale information so that the transformed images are more suitable for segmentation. Subsequently, the wavelet transform on the histograms (PDFs) removed the fluctuations so that the global local minima can be found. Finally, the adaptive threshold found by the new approach segmented the different kinds of areas of interest.

Another example is a $256 \times 256$ forward-looking infrared (FLIR) image of a tank with 256 greyscale. The original image is shown as Fig. 15(a). The $L_{h} L_{v}$ parts of its wavelet transforms from scales 1 to 4 are given in Fig. 15(b)-(e). Fig. 16 shows the PDFs of these four transformed images. We still use the image in scale 2 for segmentation and take 5-scale wavelet transforms for the histogram of the image in scale 2. They are illustrated in Fig. 17. The scale is adaptively selected to obtain local minima. Because this image is a little noisy, the unnecessary details with very low probability are abandoned when the wavelet transformed PDF curve is processed. Figs. 18(a)-20(a) show the segmentation results using local minima $\lambda_{3}>\lambda_{2}>\lambda_{1}$ as thresholds, respectively. Figs. 18(b)-20(b) show the corresponding masked images by $I_{\text {seg }} \cdot I+I$. The brightest highlight area appears in Fig. 18. The area of the tank and some other bright areas appear in Fig. 19. The largest difference between two classes, the sky and the ground as seen from the original image Fig. 15(a), is evident in Fig. 20.

In this example, it can be seen that the segmented areas contain the areas of interest. Of course, if some more details such as edges of objects are desired, other algorithms may be used in the selected areas to obtain them. Obviously, it is far more ef- 

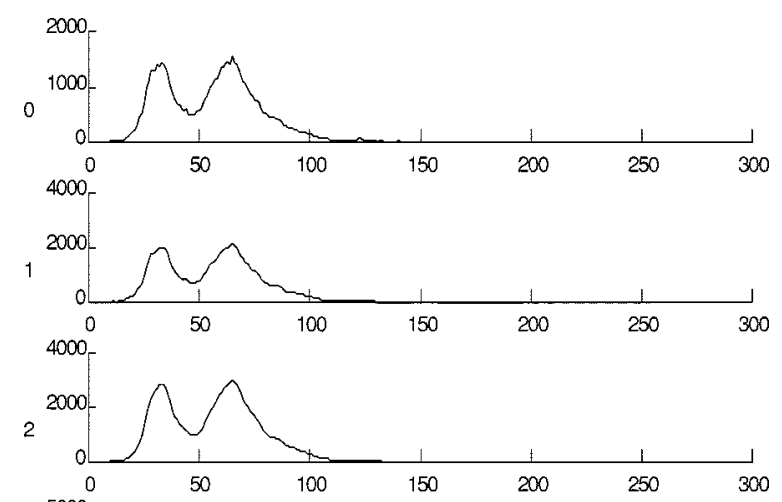

3
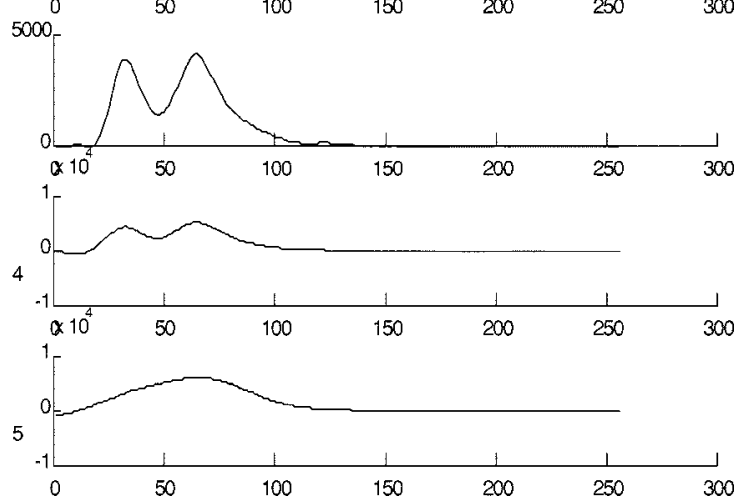

Fig. 17. Five-scale wavelet transform for the histogram of the image in scale 2 [Fig. 15(c)].

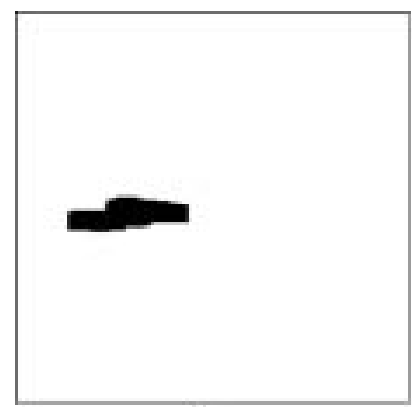

(4)

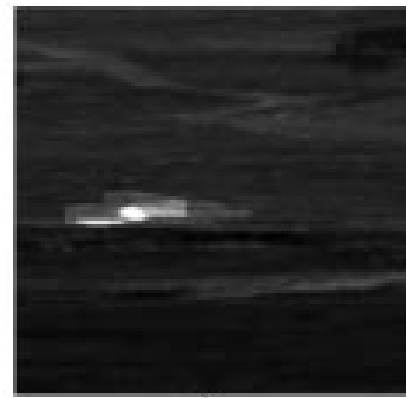

(b)
Fig. 18. (a) Segmented areas using $\lambda_{3}$. (b) Masked image using the segmented image $I_{s e g}$ in (a) by $I_{s e g} \cdot I+I$.

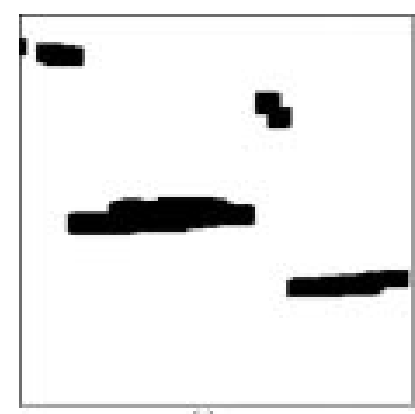

(a)

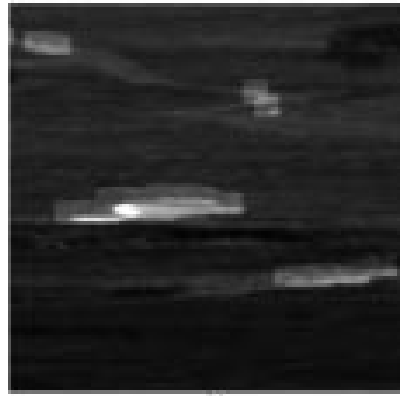

(b)
Fig. 19. (a) Segmented areas using $\lambda_{2}$. (b) Masked image using the segmented image $I_{\text {seg }}$ in (a) by $I_{s e g} \cdot I+I$.

ficient than using the algorithms in whole images. As we have indicated before, the presented method can also be iterated hierarchically and use different scales at each stage.

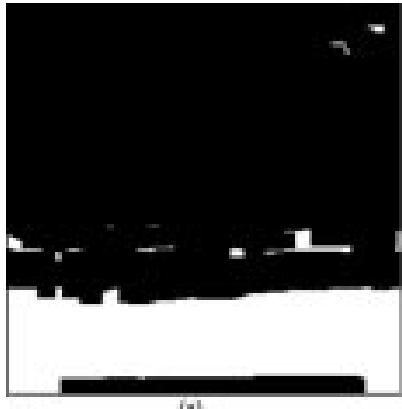

(a)

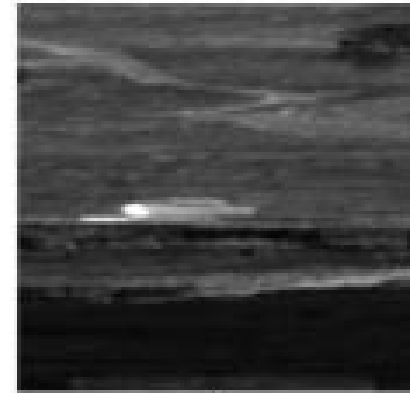

(P)
Fig. 20. (a) Segmented areas using $\lambda_{1}$. (b) Masked image using the segmented image $I_{\text {seg }}$ in (a) by $I_{\text {seg }} \cdot I+I$.

\section{CONCLUSION}

We have presented a new general method for the segmentation of bright targets in an image. Wavelet transforms are used in the new method and the Bayes classifier is employed for the segmentation problem. An approach for choosing the threshold adaptively by looking for the global local minima of the PDFs of wavelet transformed images is proposed. Based on the assumption of Gaussian distributions, the adaptive threshold by the new method is compared with the Bayes threshold. It is shown that in general practical cases, the performance of the proposed threshold is often very close to the Bayes threshold, which is the optimal threshold from the statistical point of view.

The examples show that the new method is effective to segment the tumors in mammograms and it can also be used in other segmentation applications.

To select an appropriate scale of image is an important step of this method. The general principles for choosing scaling channel along with the wavelet are presented in this paper. Because the method works under the concept of general multiscale analysis, we can expect the choices are image-dependent, however, not very image-sensitive as evident by the fact that the same parameters were used for both mammograms and FLIR images.

After the processing using the new method, other algorithms, such as boundary based approaches can also be combined with the new method depending on what is of interest in various applications.

Furthermore, although the new method is based on the distribution of the greylevel of image, it is also possible to use the method to other specific features. That is, a new image can be constructed by extracting some features from the original image for segmentation. For example, we have used it to detect the singularities, such as the microcalcifications in mammograms, and it also shows good performance [20].

\section{ACKNOWLEDGMENT}

The authors would like to thank J. Wehnes, Laboratory for Vision Systems, University of Texas, Austin, for providing the FLIR images and the anonymous reviewers for their helpful suggestions.

\section{REFERENCES}

[1] L. G. Minor and J. Sklansky, "The detection and segmentation of blobs in infrared images," IEEE Trans. Syst., Man, Cybern., vol. SMC-11, no. 3, pp. 194-201, 1981. 
[2] S. A. Dudani, B. Smithgall, and P. Robins, "Target acquisition and extraction from cluttered backgrounds," in Proc. SPIE, IR Technol. Target Detection Classification, vol. 302, 1981, pp. 43-50.

[3] D. T. Long, I. E. Abdou, and S. Sutha, "Parallel algorithm for modeldirected real-time image segmentation," in Proc. SPIE, Realtime Image Processing, vol. 302, 1990, pp. 2-7.

[4] S. M. Dunn, D. Harwood, and L. S. Davis, "Local estimation of the uniform error threshold," IEEE Trans. Pattern Anal. Machine Intell., vol. PAMI-6, no. 6, pp. 742-747, 1984.

[5] A. K. Jain, Fundamentals of Digital Image Processing. Englewood Cliffs, NJ: Prentice-Hall, 1986.

[6] P. K. Sahoo, S. Soltani, and A. K. C. Wong, "A survey of thresholding techniques," Comput. Vis. Graph. Image Process., vol. 41, pp. 233-260, 1988.

[7] J. S. Weszka and A. Rosenfeld, "Threshold evaluation techniques," IEEE Trans. Syst., Man, Cybern., vol. SMC-8, no. 8, pp. 622-629, 1978.

[8] B. Chanda and D. D. Majumder, "A note on the use of the gray-level co-occurrence matrix in threshold selection," Signal Process., vol. 15, pp. 149-167, 1988 .

[9] W.-N. Lie, "Automatic target segmentation by locally adaptive image thresholding," IEEE Trans. Image Processing, vol. 41, pp. 1036-1041, July 1995.

[10] S. Mallat, "A theory for multiresolution signal decomposition: The wavelet representation," IEEE Trans. Pattern Anal. Machine Intell., vol. 11, no. 7, pp. 674-693, July 1989.

[11] I. Daubechies, Ten Lectures on Wavelets. Philadelphia, PA: SIAM, 1992.

[12] O. Rioul, "A discrete-time multiresolution theory," IEEE Trans. Signal Processing, vol. 41, pp. 2691-2606, Aug. 1993.

[13] O. Rioul and P. Duhamel, "Fast algorithms for discrete and continuous wavelet transforms," IEEE Trans. Inform. Theory, vol. 38, pp. 569-586, Mar. 1992.

[14] M. J. Shensa, "The discrete wavelet transform: Wedding the trous and mallat algorithms," IEEE Trans. Signal Processing, vol. 40, pp. 2464-2482, Oct. 1992.

[15] A. N. Akansu and R. A. Haddad, Multiresolution Signal Decomposition. New York: Academic, 1992.

[16] A. Papoulis, Probability, Random Variables, and Stochastic Processes. New York: McGraw-Hill, 1965.

[17] H. L. Van Trees, Detection, Estimation, and Modulation Theory. Part I. New York: Wiley, 1968.
[18] J. T. Tou and R. C. Gonzalez, Pattern Recognition Principles. Reading, MA: Addison-Wesley, 1974.

[19] F. Hess, M. Kraft, M. Rivhter, and H. Bockhorn, "Comparison and assessment of various wavelet and wavelet packet based denoising algorithms for noisy data," Wavelet Dig., vol. 6, no. 2, http://www.rhrk.unikl.de/ mkraft/paper/ecmi96-paper252.ps, 1997.

[20] X.-P. Zhang, M. Desai, and R. Shah, "On processing of the microcalcifications using wavelets," Tech. Rep., submitted for publication.

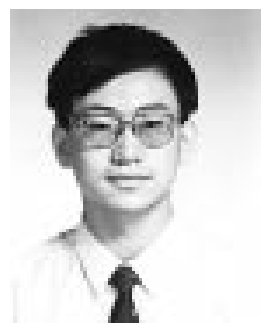

Xiao-Ping Zhang (M'97) was born in Xinjiang, China, in 1972. He received the B.S., M.S., and Ph.D. degrees from Tsinghua University, Beijing, in 1992, 1993, and 1996 respectively, all in electronic engineering.

He was a Research Associate in High-Speed Signal Processing Laboratory and VHSIC Laboratory in Tsinghua University from 1990 to 1996 . From 1996 to 1998, he was a Postdoctoral Fellow at the University of Texas, San Antonio, and then at the Beckman Institute, the University of Illinois at Urbana-Champaign. He held research and teaching positions at the Communication Research Laboratory, McMaster University, in 1999. From 1999 to 2000, he was a Senior DSP Engineer at SAM Technology, San Francisco, CA, and a Consultant at San Francisco Brain Research Institute. In 2000, he joined the Department of Electrical and Computer Engineering, Ryerson Polytechnic University, Toronto, Ont., Canada, as an Assistant Professor. His research interests include neural networks and signal processing for communications, multimedia, biomedical, and bioinfomatics applications.

Dr. Zhang received the Mei Yiqi Fellowship in 1996 from Tsinghua University. In 1994, he was a recipient of the Science and Technology Progress Award by State Education Commission of China, for his significant contribution in a National High-Tech Project.

Mita D. Desai, photograph and biography not available at time of publication. 